\title{
Photon rockets and gravitational radiation
}

\author{
Thibault Damour \\ Institut des Hautes Etudes Scientifiques \\ 91440 Bures-sur-Yvette, France \\ and \\ DARC, CNRS - Observatoire de Paris \\ 92195 Meudon, France
}

November 24, 1994

\begin{abstract}
The absence of gravitational radiation in Kinnersley's "photon rocket" solution of Einstein's equations is clarified by studying the mathematically well-defined problem of point-like photon rockets in Minkowski space (i.e. massive particles emitting null fluid anisotropically and accelerating because of the recoil). We explicitly compute the (uniquely defined) linearized retarded gravitational waves emitted by such objects, which are the coherent superposition of the gravitational waves generated by the motion of the massive point-like rocket and of those generated by the energy-momentum distribution of the photon fluid. In the special case (corresponding to Kinnersley's solution) where the anisotropy of the photon emission is purely dipolar we find that the gravitational wave amplitude generated by the energymomentum of the photons exactly cancels the usual $1 / r$ gravitational
\end{abstract}


wave amplitude generated by the accelerated motion of the rocket. More general photon anisotropies would, however, generate genuine gravitational radiation at infinity. Our explicit calculations show the compatibility between the non-radiative character of Kinnersley's solution and the currently used gravitational wave generation formalisms based on post-Minkowskian perturbation theory. 


\section{Introduction}

In 1969 Kinnersley [1] constructed an exact solution of Einstein's equations containing four arbitrary functions of time. In the literature (see e.g. [2]) this solution is referred to as a "photon rocket" and is interpreted as a "particle emitting null fluid anisotropically, and accelerating because of the recoil" [3] . Recently, Bonnor [3] pointed out a paradox of this interpretation: one would expect the presence of gravitational radiation emitted by the "accelerated particle", while, as first shown by Kinnersley [目], and recently confirmed by Bonnor [3], the spacetime described by Kinnersley's solution is asymptotically flat and (though non-stationary) contains no gravitational radiation at infinity. Technically, if, say, the "mass function" $m(s)$ is constant for $s \leq s_{0}$ and becomes smoothly variable for $s \geq s_{0}$, Kinnersley's spacetime is identical to Schwarzschild's spacetime near past null infinity, and has a (everywhere Petrov type D) Weyl tensor decreasing like $1 / r^{3}$ (in suitable, radiative, coordinates) near future null infinity, with a Ricci tensor decreasing like $1 / r^{2}$ corresponding to the energy-momentum of the outgoing photon fluid (see equations (11) and (12) of [1]).

One possible reaction to this paradox is to remark that the "interpretation" on which it is based cannot be given any rigourous meaning within the exact, curved spacetime framework of Einstein's theory. Indeed, the notion of "point particle" has no mathematical meaning in general relativity, and even if we somehow "fill in" Kinnersley's solution to deal with a regular, extended massive source, the notion of the "position" of the source (and of its "quadrupole moment") will have no unique definition, so that there will be no unambiguous way of saying that some massive object is accelerated and "must" therefore radiate at future null infinity'. We believe that these ambiguities in the interpretation of Kinnersley's solution are indeed very serious. However, we shall here tackle directly the physics behind the paradox raised by Bonnor by noticing that there is a mathematically well-defined situation in which we can self-consistently describe a recoiling photon rocket and compute the retarded gravitational waves it generates. More precisely, we consider, within the framework of special relativity, a point-like massive particle (described by a $\delta$ function on a time-like worldline) emitting null fluid anisotropically. Working rigourously within the framework of distribution theory [4], we prove in section 3 (after having introduced our notation

\footnotetext{
${ }^{1}$ Remembering that Kinnersley's solution can be taken to coincide with a static Schwarzschild spacetime before some retarded time, we do not have to worry about the possible presence of incoming gravitational radiation.
} 
for various quantities associated with a generic worldline in section 2) that the total energy-momentum tensor $T_{\mu \nu}=T_{\mu \nu}^{(\text {mass point })}+T_{\mu \nu}^{(\text {photons })}$ is everywhere conserved when the particle loses mass and recoils as expected from the fluxes of photon energy and momentum at infinity. In section 4 , we then compute explicitly the (retarded) linearized gravitational field generated by the total $T_{\mu \nu}$. We find that the amount of gravitational radiation at infinity depends on the anisotropies of multipole order $\ell \geq 2$ in the photon flux, there being a cancellation between the gravitational wave amplitude emitted by the energy-momentum distribution of the monopolar and dipolar photon flux, and the gravitational wave amplitude emitted by the accelerated massive particle. We conclude in section 5 that Bonnor's paradox] contains some truth, but that there is no incompatibility between the non-radiative character of Kinnersley's solution (with its purely dipolar photon flux anisotropy) and the consistency of the standard gravitational wave generation formalisms in general relativity, based on post-Minkowskian perturbation theory (such as a recently developed multipolar-post-Minkowskian formalism [6], [7], [8], [9], [10]). Concerns recently raised in the literature [11] about the reliability of the current predictions for the gravitational waves generated by, e.g., binary systems are therefore totally unjustified.

\section{Geometry of Minkowski worldlines}

Let $\mathcal{L}$ denote a timelike worldline $z^{\mu}(s)$, parametrized by the proper time $s$, in Minkowski space $\left(\mathbb{R}^{4}, \eta_{\mu \nu}\right)$ with $\eta_{\mu \nu} \equiv \operatorname{diag}(-1,+1,+1,+1)$. The fourvelocity along $\mathcal{L}$ is denoted $u^{\mu}(s) \equiv d z^{\mu}(s) / d s$ and satisfies $\eta_{\mu \nu} u^{\mu} u^{\nu}=-1$. To each point $x^{\mu}$ in Minkowski space one associates a unique "retarded" point $z_{R}^{\mu}\left(x^{\nu}\right)$ on $\mathcal{L}$, with parameter $s_{R}\left(x^{\mu}\right)$, defined as the intersection of $\mathcal{L}$ with the past light cone with vertex at $x^{\mu}$. In equations, $z_{R}^{\mu}(x) \equiv z^{\mu}\left(s_{R}(x)\right)$ with the function $s_{R}(x)$ defined as the only solution of

$$
\eta_{\mu \nu}\left(x^{\mu}-z^{\mu}\left[s_{R}(x)\right]\right)\left(x^{\nu}-z^{\nu}\left[s_{R}(x)\right]\right)=0
$$

such that $x^{0}-z_{R}^{0}>0$. One also introduces the null vectors

$$
\begin{aligned}
& y^{\mu}(x) \equiv x^{\mu}-z_{R}^{\mu}(x), \\
& k^{\mu}(x) \equiv \frac{x^{\mu}-z_{R}^{\mu}(x)}{\rho_{R}(x)},
\end{aligned}
$$

\footnotetext{
${ }^{2}$ Remember that a paradox is a "statement that seems to say something opposite to common sense or the truth; but which may contain a truth" [5].
} 
where

$$
\rho_{R}(x) \equiv-\left(x^{\mu}-z_{R}^{\mu}\right) u_{\mu}^{R}
$$

denotes the retarded distance between $x^{\mu}$ and the worldline $\mathcal{L}$. Here and in the following the Minkowski metric is used to raise or lower indices. Differentiating the defining equation (2.1) yields the spacetime derivative of $s_{R}(x)$,

$$
\partial_{\mu} s_{R}(x)=-\frac{y_{\mu}(x)}{\rho_{R}(x)}=-k_{\mu}(x)
$$

from which follows

$$
\begin{gathered}
\partial_{\mu} y^{\nu}=\delta_{\mu}^{\nu}+\rho_{R}^{-1} y_{\mu} u^{\nu}=\delta_{\mu}^{\nu}+k_{\mu} u^{\nu} \\
\partial_{\mu} \rho_{R}=\rho_{R}^{-1} y_{\mu}-u_{\mu}+\rho_{R}^{-1}(y \dot{u}) y_{\mu}=k_{\mu}-u_{\mu}+\rho_{R}(k \dot{u}) k_{\mu} .
\end{gathered}
$$

Here and in the following $\dot{u}^{\mu} \equiv d u^{\mu} / d s$ denotes the proper acceleration of the worldline $\mathcal{L}$ and we abbreviate Minkowski scalar products as $(a b) \equiv a_{\mu} b^{\mu} \equiv$ $\eta_{\mu \nu} a^{\mu} b^{\nu}$. It is also convenient to introduce notations for the unit spatial vector representing the direction of $k^{\mu}$ in the 3-plane orthogonal to $u^{\mu}$,

$$
k^{\mu}=u^{\mu}+n^{\mu}, \text { with }(u n)=0,(n n)=+1,
$$

and for the projection operator orthogonal to $u^{\mu}$ :

$$
\Delta_{\mu \nu}=\eta_{\mu \nu}+u_{\mu} u_{\nu}
$$

With this notation, and the results (2.4)-(2.6), we get also (suppressing the "retarded" label $R$ when there is no ambiguity)

$$
\begin{gathered}
\partial_{\mu} \rho=n_{\mu}+\rho(n \dot{u}) k_{\mu}, \\
\partial_{\mu} k_{\nu}=\rho^{-1}\left(\Delta_{\mu \nu}-n_{\mu} n_{\nu}\right)-(n \dot{u}) k_{\mu} k_{\nu} \\
\partial_{\mu} n_{\nu}=\rho^{-1}\left(\Delta_{\mu \nu}-n_{\mu} n_{\nu}\right)+k_{\mu} \dot{u}_{\nu}-(n \dot{u}) k_{\mu} k_{\nu} .
\end{gathered}
$$

As is well-known the null congruence $k^{\mu}$ is geodesic, shear-free and expanding:

$$
\begin{gathered}
k^{\nu} \partial_{\nu} k_{\mu}=0, \\
\partial_{\mu} k^{\mu}=-\square s_{R}(x)=\frac{2}{\rho}, \\
\partial^{\alpha} k_{\mu} \partial_{\alpha} k_{\nu}=\rho^{-2}\left[\Delta_{\mu \nu}-n_{\mu} n_{\nu}\right],
\end{gathered}
$$




$$
\partial^{\mu} k^{\nu} \partial_{\mu} k_{\nu}=2 \rho^{-2}=\frac{1}{2}\left(\partial_{\mu} k^{\mu}\right)^{2} .
$$

Finally, let us note that we can introduce "retarded" polar coordinates centered on the worldline $\mathcal{L}$, say $(s, \rho, \theta, \varphi)$, such that the polar coordinates of $x^{\mu}$ are $s_{R}(x), \rho_{R}(x)$ and two angles parametrizing the unit spatial vector $n^{\mu}(x)$, chosen, say, so as to induce the standard metric $\rho^{2}\left(d \theta^{2}+\sin ^{2} \theta d \varphi^{2}\right)$ on the 2 -spheres $s=$ const, $\rho=$ const. This still leaves a large freedom in the rotation rate of the basis vectors $\partial n^{\mu} / \partial \theta,(\sin \theta)^{-1} \partial n^{\mu} / \partial \varphi$. We shall not need to restrict this freedom. One finds that the Minkowski volume element takes (independently of the acceleration of the worldline) the familiar form

$$
d^{4} x=\rho^{2} d s d \rho d \Omega_{\mathbf{n}}
$$

with $d \Omega_{\mathbf{n}}=\sin \theta d \theta d \varphi$, while the surface element of the retarded tubes $\rho=$ const. reads

$$
d S_{\mu}=\partial_{\mu} \rho_{R} \rho_{R}^{2} d s d \Omega_{\mathbf{n}}
$$

\section{Photon rockets in Minkowski space}

A physical photon rocket would be an extended massive object emitting (in the WKB approximation) null fluid anisotropically. Let us prove that one can consistently define, in Minkowski space, the point-like limit of such an object. We consider an energy-momentum distribution which is partly concentrated as a $\delta$-distribution on a time-like worldline $\mathcal{L}$, and partly distributed as a null fluid spurting out of $\mathcal{L}$. Say

$$
T^{\mu \nu}(x)=T_{(m)}^{\mu \nu}(x)+T_{(p)}^{\mu \nu}(x),
$$

with a "matter" part

$$
T_{(m)}^{\mu \nu}(x)=\int d s m(s) u^{\mu}(s) u^{\nu}(s) \delta_{4}\left(x^{\mu}-z^{\mu}(s)\right),
$$

and a "photon" part

$$
T_{(p)}^{\mu \nu}(x)=\frac{\varepsilon\left(s_{R}(x), n^{\lambda}(x)\right)}{4 \pi \rho_{R}^{2}(x)} k^{\mu}(x) k^{\nu}(x) .
$$

It has been verified by Kinnersley [1] and Bonnor [3] that the energymomentum tensor (3.3) entails an outgoing flux of energy and momentum at infinity. They then argued heuristically that this must be compensated by 
a loss of mass $d m(s) / d s$, and a recoil $d u^{\mu} / d s$ of the "central particle". Let us verify this more rigourously by studying whether the total energy tensor $T^{\mu \nu}(x)$ can be everywhere conserved, including on $\mathcal{L}$. This will prove the consistency of the point-like limit. We shall consistently work in the framework of distribution theory, with $T^{\mu \nu}(x)$ considered as a linear functional acting on smooth, compact-support functions in $\mathbb{R}^{4}:\left\langle T^{\mu \nu}, \varphi\right\rangle=\int d^{4} x T^{\mu \nu}(x) \varphi(x)$. For instance, $\left\langle T_{(m)}^{\mu \nu}, \varphi\right\rangle=\int d s m(s) u^{\mu} u^{\nu} \varphi(z(s))$, while the distribution $T_{(p)}^{\mu \nu}(x)$ is uniquely defined from the function (3.3), in spite of its singular behaviour on the central worldline $\mathcal{L}$, because $T_{(p)}^{\mu \nu}$ is locally integrable $\left[O\left(\rho^{-2}\right)\right.$ singularity only as $\rho \rightarrow 0]$. The distributional derivative of the matter part (3.2) is easily verified to give

$$
\partial_{\nu} T_{(m)}^{\mu \nu}(x)=+\int d s \frac{d}{d s}\left(m(s) u^{\mu}\right) \delta_{4}(x-z(s)) .
$$

The computation of $\partial_{\nu} T_{(p)}^{\mu \nu}$ is more tricky. It cannot be directly done on the function (3.3) because $\partial_{\nu} T_{(p)}^{\mu \nu}(x)$ has a non locally integrable singularity, $O\left(\rho^{-3}\right)$, along $\mathcal{L}$. One way to do it is to apply the distributional definition, $\left\langle T_{(p)}^{\mu \nu},-\partial_{\nu} \varphi\right\rangle$, and use Stokes formula, with equation (2.12), when integrating by parts on a domain $\rho_{R}(x) \geq \epsilon$ before letting $\epsilon \rightarrow 0$. A more convenient technique, which is quite standard in distribution theory for functions having power-law singularities (see e.g. 歫), is to introduce a complex parameter $B$ and to work with the analytic continuation of integrals of ordinary functions. For instance, we can multiply equation (3.3) by $\rho^{B}$ and compute $\partial_{\nu}\left(\rho^{B} T_{(p)}^{\mu \nu}(x)\right)$ which is a locally integrable function for $\operatorname{Re}(B)>0$. The looked for distribution is then obtained by analytically continuing $B$ to zero. Using the derivatives recalled in section 2 , this gives after an easy calculation

$$
\partial_{\nu}\left(\rho^{B} T_{(p)}^{\mu \nu}\right)=B \rho^{B-3} \frac{\varepsilon\left(s_{R}, n^{\lambda}\right)}{4 \pi} k^{\mu} .
$$

The problem is then reduced to evaluating the analytic continuation down to $B=0$ of the four-dimensional integral

$$
I(B)=\int d^{4} x \partial_{\nu}\left(\rho^{B} T_{(p)}^{\mu \nu}\right) \varphi(x)=\frac{B}{4 \pi} \int d s d \rho d \Omega_{\mathbf{n}} \rho^{B-1} \varepsilon(s, n) k^{\mu} \varphi(x),
$$

where we have used retarded polar coordinates and equation (2.11). The $B$ factor in front shows that only an arbitrarily small neighbourhood of $\mathcal{L}$, say $\rho \leq \rho_{0}$, matters. Using the Taylor expansion of $\varphi(x)$ gives $\varphi[x(s, \rho, \theta, \varphi)]=$ $\varphi[z(s)]+O(\rho)$ when $\rho \rightarrow 0$. As the analytic continuation of $B \int_{0}^{\rho_{0}} d \rho \rho^{B-1} \rho=$ 
$B \rho_{0}^{B+1} /(B+1)$ is zero, we see that only the value of $\varphi$ along $\mathcal{L}, \varphi[z(s)]$, contributes. The integration over the angles introduces the quantity

$$
\frac{1}{4 \pi} \int d \Omega_{\mathbf{n}} \varepsilon(s, \mathbf{n}) k^{\mu}=\varepsilon_{0}(s) u^{\mu}+\varepsilon_{1}^{\mu}(s)
$$

with

$$
\begin{gathered}
\varepsilon_{0}(s) \equiv \frac{1}{4 \pi} \int d \Omega_{\mathbf{n}} \varepsilon(s, \mathbf{n}), \\
\varepsilon_{1}^{\mu}(s) \equiv \frac{1}{4 \pi} \int d \Omega_{\mathbf{n}} n^{\mu} \varepsilon(s, \mathbf{n}),
\end{gathered}
$$

while the integration over $\rho$ gives, modulo terms that tend to zero when $B$ is analytically continued to zero, $B \int_{0}^{\rho_{0}} d \rho \rho^{B-1}=\rho_{0}^{B}=e^{B \ln \rho_{0}}$, which tends to one when $B$ is continued down to zero, independently of $\rho_{0}$. Finally, this gives

$$
I(0)=\left\langle\partial_{\nu} T_{(p)}^{\mu \nu}, \varphi\right\rangle=\int d s\left(\varepsilon_{0}(s) u^{\mu}+\varepsilon_{1}^{\mu}(s)\right) \varphi[z(s)],
$$

which means that we have proven the distributional result

$$
\partial_{\nu} T_{(p)}^{\mu \nu}(x)=\int d s\left(\varepsilon_{0}(s) u^{\mu}+\varepsilon_{1}^{\mu}(s)\right) \delta_{4}(x-z(s)) .
$$

Combining (3.11) with (3.4), we conclude that the total energy-momentum tensor (3.1) will be everywhere conserved in the sense of distribution theory, $\left\langle\partial_{\nu} T^{\mu \nu}, \varphi\right\rangle=0$, if and only if

$$
\frac{d}{d s}\left(m(s) u^{\mu}(s)\right)+\varepsilon_{0}(s) u^{\mu}+\varepsilon_{1}^{\mu}(s)=0 .
$$

Noting that $u_{\mu} \varepsilon_{1}^{\mu}=0$ from its definition (3.9), equation (3.12) is equivalent to

$$
\begin{gathered}
\dot{m}=-\varepsilon_{0}, \\
m \dot{u}^{\mu}=-\varepsilon_{1}^{\mu} .
\end{gathered}
$$

These are the results of [3] obtained here without relying on a heuristic energy-momentum balance argument.

\section{Linearized gravitational waves emitted by photon rockets}

The conserved energy-momentum tensor (3.1) can be consistently taken as distributional source term for linearized gravity. The linearized Einstein 
equations for $g_{\mu \nu}=\eta_{\mu \nu}+h_{\mu \nu}$ (or the field equations of the massless spin-2 field $\left.h_{\mu \nu}\right)$ read

$$
\square h_{\mu \nu}+\partial_{\mu \nu} h-\partial_{\mu \alpha} h_{\nu}^{\alpha}-\partial_{\nu \alpha} h_{\mu}^{\alpha}=-16 \pi G\left(T_{\mu \nu}-\frac{1}{2} \eta_{\mu \nu} T\right),
$$

where $\square \equiv \eta^{\alpha \beta} \partial_{\alpha \beta}, h \equiv h_{\alpha}^{\alpha}, T \equiv T_{\alpha}^{\alpha}$, indices being raised and lowered by the Minkowski metric. To make contact with usual perturbation techniques in general relativity let us start by looking for solutions of equation (4.1) in harmonic gauge

$$
\partial^{\nu} h_{\mu \nu}^{(\mathrm{har})}-\frac{1}{2} \partial_{\mu} h^{(\mathrm{har})}=0
$$

This yields as usual

$$
h_{\mu \nu}^{(\mathrm{har})}=-16 \pi G\left(T_{\mu \nu}-\frac{1}{2} \eta_{\mu \nu} T\right)
$$

To simplify the discussion we assume that the photon rocket started (smoothly) its activity only at some finite time in the past, i.e. $\varepsilon\left(s, n^{\mu}\right)=0$ for $s \leq s_{0}$. This implies that $m(s)$ was constant and $\mathcal{L}$ straight before $s_{0}$ (see equations (3.13)), and that the non compact-support part of $T^{\mu \nu}(x)$, namely $T_{(p)}^{\mu \nu}(x)$, equation (3.3), has its support entirely contained in the forward light cone with vertex $z^{\mu}\left(s_{0}\right)$. These properties ensure that the retarded solution of (4.3), i.e. the convolution of the retarded Green's function,

$$
G_{R}\left(x^{\mu}\right) \equiv-\frac{1}{2 \pi} \theta\left(x^{0}\right) \delta\left(\eta_{\mu \nu} x^{\mu} x^{\nu}\right) \equiv-\frac{1}{4 \pi} \frac{\delta\left(x^{0}-|\mathbf{x}|\right)}{|\mathbf{x}|} ; \square G_{R}(x)=\delta_{4}(x)
$$

with the right-hand side of (4.3), is mathematically well-defined [3, and can be characterized as being the only solution of (4.3) containing no incoming radiation. We write it as

$$
h_{\mu \nu}^{(\mathrm{har})}(x)=-16 \pi G \square_{R}^{-1}\left(T_{\mu \nu}-\frac{1}{2} \eta_{\mu \nu} T\right),
$$

where $\left(\square_{R}^{-1} S\right)(x) \equiv \int d^{4} y G_{R}(x-y) S(y)$ denotes the retarded potential operator.

The linear split (3.1) of $T_{\mu \nu}$ leads to a corresponding linear split of $h_{\mu \nu}^{(\mathrm{har})}$, say

$$
h_{\mu \nu}^{(\mathrm{har})}(x)=h_{\mu \nu}^{(m)}(x)+h_{\mu \nu}^{(p)}(x) .
$$

\footnotetext{
${ }^{3}$ In particular, it is given by a compact-support three-dimensional integral; the "bad" $\sim 1 / r^{2}$ behaviour of the source term at future null infinity causing no convergence problem in the retarded potential integral.
} 
The "matter" part $h_{\mu \nu}^{(m)}$ is obtained in a standard way from its source (3.2) using the covariant form of $G_{R}\left(x^{\mu}-z^{\mu}(s)\right) \propto \delta\left[\left(x_{\mu}-z_{\mu}(s)\right)\left(x^{\mu}-z^{\mu}(s)\right)\right]$ and the formula $\delta[\varphi(s)]=\sum_{n} \delta\left(s-s_{n}\right) /\left|\varphi^{\prime}\left(s_{n}\right)\right|$ where $n$ labels the roots $s_{n}$ of $\varphi(s)=0$ :

$$
h_{\mu \nu}^{(m)}(x)=2 G\left(\frac{m(s)\left[2 u_{\mu} u_{\nu}+\eta_{\mu \nu}\right]}{\rho}\right)_{R} .
$$

Here, as in section 2 , the suffix $R$ indicates that all $s$-dependent quantities must be evaluated at the retarded point $s_{R}(x)$ (Liénard-Wiechert potential).

The "photon" part $h_{\mu \nu}^{(p)}$ is given by the following retarded potential integral

$$
h_{\mu \nu}^{(p)}(x)=-4 G \square_{R}^{-1}\left(\frac{\varepsilon\left(s_{R}, n\right) k_{\mu} k_{\nu}}{\rho_{R}^{2}}\right),
$$

which is well-defined as the integral of a function (rather than a distribution) because of the local integrability of $1 / \rho^{2}$. The integral (4.8) can be analytically reduced to a one-dimensional integral over the proper time $s$ (on the domain $s \leq s_{R}(x)$ ) by using the formula (2.24) of Ref. [10] However, a more convenient way of studying $h_{\mu \nu}^{(p)}$ is, following the study of the $u^{\alpha \beta}$ term in Ref. [10], which has the same structure as $h_{\mu \nu}^{(p)}$ (for a straight worldline), to introduce a simplifying gauge transformation. The role of this gauge transformation (or linearized coordinate transformation) is to transform the source $\propto k_{\mu} k_{\nu} / \rho^{2}$ in equation (4.8) into a source proportional to $1 / \rho^{3}$ which turns out to be much simpler to deal with tackle non locally integrable terms $\sim \rho^{-3}$. This situation is technically very similar to what happened in section 3 where we needed to evaluate the distributional derivative of $T_{(p)}^{\mu \nu} \sim \rho^{-2}$, i.e. to make sense of integrals involving $\partial_{\lambda} T_{(p)}^{\mu \nu} \sim \rho^{-3}$. Like in section 3 , a technically convenient, and mathematically rigourous, way of dealing with such terms is to introduce a complex parameter $B$ and to work with the analytic continuation of certain integrals.

Given a function $\sigma\left(s, n^{\mu}\right)$ which reduces to an angle-independent constant $\sigma_{0}$ for $s \leq s_{0}$ (i.e. when $\varepsilon\left(s, n^{\mu}\right)$ vanishes) and which will be related to $m(s)$

\footnotetext{
${ }^{4}$ To generalize the result of [10] which assumes a straight worldline $\mathcal{L}$ to the present case of a curved worldline, one needs (beyond multipole-expanding $\varepsilon(n)$ ) to represent the source term in (4.8) as a one-dimensional integral of elementary contributions with support on light cones with vertex on $\mathcal{L}$.

${ }^{5}$ Another utility of that gauge transformation is to deal away with the terms of order $(\ln \rho) / \rho$ in $h_{\mu \nu}^{(\text {har) }}$ at future null infinity. As is well-known these terms, due to the slow $1 / \rho^{2}$ fall off of $T_{\mu \nu}$, arise in harmonic coordinates but can be gauged away by introducing some suitable, "radiative" coordinates.
} 
and $\varepsilon\left(s, n^{\mu}\right)$ below, we define the gauge transformation $\xi_{\mu}(x)$ as follows. We first consider the retarded potential integral

$$
\xi_{\mu}^{B}(x) \equiv \square_{R}^{-1}\left(\frac{1}{2} \rho_{R}^{B-2} \sigma\left(s_{R}(x), n\right) k_{\mu}\right),
$$

which depends on the complex parameter $B$, and is convergent when $-1<$ $\operatorname{Re}(B)<0$. Then we analytically continue $\xi_{\mu}^{B}(x)$ in $B$. Generalizing standard arguments (see, e.g., Refs [6], [7]) $\xi_{\mu}^{B}(x)$ is easily seen to admit a continuation as a meromorphic function over the whole complex $B$ plane. Denoting by $F P_{B=0}$ ("Finite Part at $B=0$ ") the operation of taking the constant term (zeroth power of $B$ ) in the Laurent expansion of $\xi_{\mu}^{B}(x)$ around $B=0$, we then define

$$
\xi_{\mu}(x) \equiv F P_{B=0}\left(\xi_{\mu}^{B}(x)\right) .
$$

As $\rho^{-2}$ is locally integrable the analytic continuation factor $\rho^{B}$ is not needed in (4.9a) to deal with the neighbourhood of $\mathcal{L}$, but is useful to deal with the slow fall-off $\left(\sim \rho^{-2}\right)$ at past null infinity due to the fact that $\sigma$ becomes a non-zero constant in the past. The retarded integral $(4.9 \mathrm{a})$ is convergent when $-1<\operatorname{Re}(B)<0$, and is easily seen to have a simple pole at $B=0$ when analytically continued upwards in $\operatorname{Re}(B)$. The effect of this pole is to introduce a term proportional to $\sigma_{0} u_{\mu}^{0} \rho_{0}^{B} / B$ in $\xi_{\mu}^{B}$, and therefore, using the Laurent expansion, $B^{-1} \rho_{0}^{B}=B^{-1} \exp \left(B \ln \rho_{0}\right)=B^{-1}+\ln \rho_{0}+O(B)$, a term proportional to $\sigma_{0} u_{\mu}^{0} \ln \rho_{0}$ in $\xi_{\mu}$. [Here, $\rho_{0}$ denotes the spatial distance between the field point $x^{\mu}$ and the incoming straight worldline with fourvelocity $u_{\mu}^{0}$. This logarithmic term disappears when taking $x$-derivatives, which corresponds to the fact that the $B$-dependent integrals considered below will have a better convergence at past null infinity, and generate no poles at $B=0$.

Thanks to the properties of analytically continued integrals we can commute $x$-derivatives with the integral operator $\square_{R}^{-1}$. [When dealing with an integral such as (4.9a), the integrand of which has power-law singularities both on $\mathcal{L}$ and at past null infinity, it is convenient to split the spatial domain of integration in two pieces, one of them being compact and centered on $\mathcal{L}$; see, e.g., section 3 of [7] for a similar situation.] This yields

$$
\partial_{\mu} \xi_{\nu}^{B}=\frac{1}{2} \square_{R}^{-1}\left[\partial_{\mu}\left(\rho^{B-2} \sigma k_{\nu}\right)\right]
$$

where, using the results of section 2 ,

$$
\partial_{\mu}\left(\rho_{R}^{B-2} \sigma\left(s_{R}, n^{\alpha}\right) k_{\nu}\right)=-\rho_{R}^{B-2} D \sigma k_{\mu} k_{\nu}
$$




$$
\begin{gathered}
+\rho_{R}^{B-3}\left[\sigma\left\{\Delta_{\mu \nu}+(B-2) n_{\mu} u_{\nu}+(B-3) n_{\mu} n_{\nu}\right\}\right. \\
\left.+\frac{\partial \sigma}{\partial n_{\alpha}}\left(\Delta_{\alpha \mu}-n_{\alpha} n_{\mu}\right) k_{\nu}\right] .
\end{gathered}
$$

The quantity $D \sigma$ in (4.11) denotes the action of a certain first-order differential operator on $\sigma\left(s, n^{\alpha}\right)$ :

$$
D \sigma\left(s, n^{\alpha}\right)=\frac{\partial \sigma}{\partial s}+\left[(n \dot{u}) k_{\alpha}-\dot{u}_{\alpha}\right] \frac{\partial \sigma}{\partial n_{\alpha}}+(3-B)(n \dot{u}) \sigma .
$$

Here we consider $\sigma\left(s, n^{\alpha}\right)$ as some explicit function of $s$ and the four components of $n^{\alpha}$, e.g. the one obtained by expanding the angular dependence of $\sigma$ in cartesian symmetric-trace-free $(\mathrm{STF})$ polynomials in $n^{\alpha}$ [equivalent to a spherical harmonic expansion in $Y_{\ell}^{m}(\theta, \varphi)$ ]

$$
\sigma\left(s, n^{\alpha}\right)=\sum_{\ell=0}^{\infty} \sigma_{\alpha_{1} \alpha_{2} \ldots \alpha_{\ell}}(s) \widehat{n}^{\alpha_{1} \alpha_{2} \ldots \alpha_{\ell}},
$$

where $\widehat{n}^{\alpha_{1} \ldots \alpha_{\ell}}$ denotes the STF projection (within the spatial hyperplane orthogonal to $u^{\alpha}$ ) of $n^{\alpha_{1}} n^{\alpha_{2}} \ldots n^{\alpha_{\ell}}$. For instance, $\widehat{n}^{\mu \nu} \equiv n^{\mu} n^{\nu}-\frac{1}{3} \Delta^{\mu \nu}$. Without loss of generality, we can also consider that the $s$-dependent coefficients in (4.13) are purely spatial $\left(u^{\alpha_{1}} \sigma_{\alpha_{1} \ldots \alpha_{\ell}}=\ldots=u^{\alpha_{\ell}} \sigma_{\alpha_{1} \ldots \alpha_{\ell}}=0\right)$ and STF, in which case we can replace $\widehat{n}^{\alpha_{1} \ldots \alpha_{\ell}}$ by $n^{\alpha_{1}} \ldots n^{\alpha_{\ell}}$ in (4.13) so that the $s$ derivative in (4.12) acts only on $\sigma_{\alpha_{1} \ldots \alpha_{\ell}}(s)$. Evidently, the quantities $D \sigma$ and $\partial \sigma / \partial n_{\alpha}\left(\Delta_{\alpha \mu}-n_{\alpha} n_{\mu}\right)$ entering (4.11) are defined independently of the multipole expansion (4.13), but an alternative definition would imply choosing some explicit parametrization of $n^{\alpha}$ in terms of two polar angles $\theta, \varphi$ (i.e. choosing a triad of vectors orthogonal to $u^{\alpha}$ ) which we prefer to shun doing.

We see from equation (4.11) that the gauge transformation $h_{\mu \nu}^{\prime}=h_{\mu \nu}+$ $\partial_{\mu} \xi_{\nu}+\partial_{\nu} \xi_{\mu}$ generates terms proportional to $k_{\mu} k_{\nu} / \rho^{2}$ which can cancel the original "photon" piece (4.8) and replace it by the retarded potential of source terms $\propto \rho^{-3}$. To perform this transformation explicitly, it is convenient to consider that the photon flux function $\varepsilon\left(s, n^{\alpha}\right)$ is, similarly to equation (4.13), decomposed in angular multipoles

$$
\varepsilon\left(s, n^{\alpha}\right)=\sum_{\ell=0}^{\infty} \varepsilon_{\ell}\left(s, n^{\alpha}\right)
$$

where $\varepsilon_{\ell}\left(n^{\alpha}\right)$ is a linear combination of $\widehat{n}^{\alpha_{1} \ldots \alpha_{\ell}}$. The first two terms in the multipole expansion (4.14), namely the monopole $\varepsilon_{0}$ and dipole parts $\varepsilon_{1}$, are 
fully determined by the integrals (3.8) and (3.9). More precisely $\varepsilon_{0}$ (defined by equation (4.14) as the monopole piece of $\varepsilon(s, n))$ is identical with $\varepsilon_{0}(s)$ defined by equation (3.8), while the dipole piece of $\varepsilon(s, n)$ reads

$$
\varepsilon_{1}\left(s, n^{\alpha}\right)=3 \varepsilon_{1}^{\mu}(s) n_{\mu}
$$

in terms of the definition (3.9).

Let us first consider the special case (that we shall the "Kinnersley case") where the photon flux $\varepsilon(s, n)$ contains only a monopole and a dipole piece:

$$
\varepsilon^{K}\left(s, n^{\alpha}\right)=\varepsilon_{0}(s)+3 \varepsilon_{1}^{\alpha}(s) n_{\alpha} .
$$

In view of the balance equations (3.13), this can be rewritten in terms of "mechanical" quantities

$$
\varepsilon^{K}\left(s, n^{\alpha}\right)=-[\dot{m}(s)+3 m(s)(\dot{u} n)] .
$$

Let us correspondingly consider the special case where $\sigma$ is purely monopolar: $\sigma(s, n)=\sigma^{K}(s)$. From equations (4.10)-(4.12) (which simplify when $\sigma$ depends only on $s$ ) we find for the gauge transformation

$$
\begin{gathered}
\partial_{\mu} \xi_{\nu}^{B(K)}+\partial_{\nu} \xi_{\mu}^{B(K)}=\square_{R}^{-1}\left\{-\rho^{B-2}\left[\dot{\sigma}^{K}+(3-B) \sigma^{K}(n \dot{u})\right] k_{\mu} k_{\nu}\right. \\
\left.+\rho^{B-3} \sigma^{K}\left[\frac{B}{3} \Delta_{\mu \nu}+\frac{B-2}{2}\left(n_{\mu} u_{\nu}+n_{\nu} u_{\mu}\right)+(B-3) \widehat{n}_{\mu \nu}\right]\right\},
\end{gathered}
$$

where we have decomposed the functions of $n^{\alpha}$ in irreducible multipoles of order 0,1 and $2\left(\widehat{n}^{\mu \nu}=n^{\mu} n^{\nu}-\frac{1}{3} \Delta^{\mu \nu}\right)$.

Comparing (4.18) with the result of inserting equation (4.17) into equation (4.8) we are led to defining

$$
\sigma^{K}(s) \equiv+4 G m(s)
$$

For this choice we see easily that the first term on the right-hand side of (4.18) is analytic at $B=0$ (no pole; thanks to the vanishing of $\dot{\sigma}^{K}$ and $\sigma^{K}(n \dot{u})$ in the past) and that its value at $B=0$ is

$$
+4 G \square_{R}^{-1}\left(\rho^{-2} \varepsilon^{K}\left(s_{R}, n\right) k_{\mu} k_{\nu}\right)
$$

which is precisely the opposite of $h_{\mu \nu}^{(p)}(x)$, defined in equation (4.8), in the Kinnersley case. 
We are then left with evaluating three types of terms: "monopolar" terms of the form $\square_{R}^{-1}\left(B \rho^{B-3} \varphi(s)\right)$, and "dipolar" and "quadrupolar" terms of the form $\square_{R}^{-1}\left(\rho^{B-3} \varphi(s) \widehat{n}_{\alpha_{1} \ldots \alpha_{\ell}}\right)$ with $\ell=1$ or 2 . The monopolar term

$$
\square_{R}^{-1}\left(\frac{B}{3} \rho_{R}^{B-3} \sigma^{K}\left(s_{R}\right) \Delta_{\mu \nu}\left(s_{R}\right)\right)
$$

appearing in the second square bracket on the right-hand side of (4.18) is easily evaluated, when $B$ is analytically continued to zero, by the same reasoning used in section 3 above to deal with the integral of the product of the right-hand side of equation (3.5) with a smooth function [here that smooth function is $G_{R}\left(x-x^{\prime}\right)$ ]. Denoting $A C_{B=0}$ the operation of taking the analytic continuation at $B=0$, we deduce from the results of section 3

$$
A C_{B=0}\left\{B \rho^{B-3} \varphi(s)\right\}=4 \pi \int d s \varphi(s) \delta_{4}(x-z(s))
$$

in the sense of distribution theory [where the $B$ factor in front ensures the absence of pole at $B=0]$. Convoluting the Green's function (4.4) with (4.21), we find that the analytic continuation of the monopolar term (4.20) yields

$$
-\frac{1}{3} \frac{\sigma^{K}\left(s_{R}\right) \Delta_{\mu \nu}\left(s_{R}\right)}{\rho_{R}}=-\frac{4 G}{3}\left(\frac{m(s) \Delta_{\mu \nu}}{\rho}\right)_{R} .
$$

The dipolar and quadrupolar terms (last two terms on the right-hand side of equation (4.18)) can be evaluated by using explicit formulas derived in Ref. [7]. There, indeed, it was shown that, in the case of a straight central worldline $\mathcal{L}_{0}$, the analytic continuation at $B=0$ of the retarded potential generated by $r^{B-k} H(t-r) \widehat{n}_{i_{1} \ldots i_{\ell}}$ was expressible, when $3 \leq k \leq \ell+2$, as a finite sum of derivatives of $H(t-r)$ divided by powers of $r$. In particular, when $k=3, \ell \geq 1$ and $H(t)$ is the one-dimensional delta function $\delta(t-\sigma)$, equation (4.24) of Ref. [7] reads

$$
\begin{gathered}
A C_{B=0}\left\{\square_{R}^{-1}\left(r^{B-3} \delta(t-r-\sigma) \widehat{n}_{i_{1} \ldots i_{\ell}}\right)\right\}= \\
-\frac{1}{\ell(\ell+1)} \frac{\delta(t-r-\sigma)}{r} \widehat{n}_{i_{1} \ldots i_{\ell}} ;(\ell \geq 1) .
\end{gathered}
$$

Here, the central straight worldline $\mathcal{L}_{0}$ is taken as time axis, $r$ denotes the spatial distance between the field point $\left(x^{\mu}\right)=\left(t, x^{i}\right)$ and $\mathcal{L}_{0}, n^{i} \equiv x^{i} / r$, and $\sigma$ is a constant, parametrizing a specific point $(\sigma, \mathbf{0})$ on $\mathcal{L}_{0}$. 
Equation (4.23) can be generalized to the case, of interest for the present paper, of a generic curved worldline $\mathcal{L}$ by noticing that if $\mathcal{L}_{0}$ is taken to be the tangent to $\mathcal{L}$ at $z^{\mu}(\sigma)$ then $[\delta(t-r-\sigma)]_{\mathcal{L}_{0}}=\left[\delta\left(s_{R}(x)-\sigma\right)\right]_{\mathcal{L}}$ as distributions in $\mathbb{R}^{4}$. [Indeed, both distributions have the future light cone of $z^{\mu}(\sigma)$ as support, and using the fact that the volume element in retarded coordinates (2.11) does not depend on the acceleration $\dot{u}^{\mu}$ one sees immediately that $\left.\int d^{4} x \delta(t-r-\sigma) \varphi(x)=\int d^{4} x \delta\left(s_{R}(x)-\sigma\right) \varphi(x)\right]$. Having generalized (4.23) to an elementary source with support on a light cone centered on $\mathcal{L}$, we can pass to a general retarded-time-dependence of the source by integrating over the parameter $\sigma$ using the decomposition $H\left(s_{R}(x)\right)=\int d \sigma H(\sigma) \delta\left(s_{R}(x)-\sigma\right)$. This yields

$$
\begin{gathered}
A C_{B=0}\left\{\square_{R}^{-1}\left(\rho_{R}^{B-3} H\left(s_{R}(x)\right) \widehat{n}_{\mu_{1} \ldots \mu_{\ell}}\right)\right\}= \\
-\frac{1}{\ell(\ell+1)} \frac{H\left(s_{R}(x)\right)}{\rho_{R}} \widehat{n}_{\mu_{1} \ldots \mu_{\ell}} ;(\ell \geq 1) .
\end{gathered}
$$

It is remarkable that, though the left-hand side, evaluated at the field point $x^{\mu}$, is an integral over the past light cone of $x^{\mu}$ whose integrand depends on the full past history of the source, i.e. on the value of the function $H(s)$ over the interval $-\infty \leq s \leq s_{R}(x)$, the final result on the right-hand side depends only on the value of $H(s)$ at the retarded point $s_{R}(x)$. This simplification does not hold for integrands proportional to $\rho_{R}^{-2}$, as they appeared in the original expression (4.8) before introducing the gauge transformation (4.9).

Using the result (4.24) we can read off equation (4.18) the contributions of the dipolar and quadrupolar terms in the last bracket. Namely:

$$
\begin{gathered}
\frac{\sigma^{K}}{\rho_{R}}\left[+\frac{1}{2}\left(n_{\mu} u_{\nu}+n_{\nu} u_{\mu}\right)+\frac{1}{2} \widehat{n}_{\mu \nu}\right]= \\
2 G \frac{m\left(s_{R}\right)}{\rho_{R}}\left[n_{\mu} u_{\nu}+n_{\nu} u_{\mu}+\widehat{n}_{\mu \nu}\right] .
\end{gathered}
$$

Gathering our results, we conclude that the gauge-transform of the original harmonic linearized field (4.6),

$$
h_{\mu \nu}^{\prime}(x)=h_{\mu \nu}^{(\mathrm{har})}(x)+\partial_{\mu} \xi_{\nu}+\partial_{\nu} \xi_{\mu}=h_{\mu \nu}^{(m)}(x)+h_{\mu \nu}^{(p)^{\prime}}(x),
$$

with

$$
h_{\mu \nu}^{(p)^{\prime}}(x) \equiv h_{\mu \nu}^{(p)}(x)+\partial_{\mu} \xi_{\nu}+\partial_{\nu} \xi_{\mu},
$$

is given, in the Kinnersley case (4.16), (4.17), by adding the matter contribution (4.7) and the gauge-transformed photon one obtained by adding (4.22) 
and (4.25):

$$
\left[h_{\mu \nu}^{(p)^{\prime}}\right]^{K}=2 G \frac{m\left(s_{R}\right)}{\rho_{R}}\left[-\frac{2}{3} \Delta_{\mu \nu}+n_{\mu} u_{\nu}+n_{\nu} u_{\mu}+\widehat{n}_{\mu \nu}\right]_{R} .
$$

Replacing the definitions $\widehat{n}_{\mu \nu} \equiv n_{\mu} n_{\nu}-\frac{1}{3} \Delta_{\mu \nu}, \Delta_{\mu \nu}=\eta_{\mu \nu}+u_{\mu} u_{\nu}$ and eliminating $n_{\mu}$ in favour of $k_{\mu} \equiv u_{\mu}+n_{\mu}$, the result (4.28) can be rewritten as

$$
\left[h_{\mu \nu}^{(p)^{\prime}}\right]^{K}=2 G \frac{m\left(s_{R}\right)}{\rho_{R}}\left[-\eta_{\mu \nu}-2 u_{\mu} u_{\nu}+k_{\mu} k_{\nu}\right]_{R} .
$$

We recognize in the first two terms of the (gauge-transformed) photon contribution (4.29) the opposite of the matter contribution (4.7). We have therefore proven by an explicit computation that, after a suitable gauge transformation (or linearized coordinate transformation) (4.9), there were terms in the gravitational field generated by the photon energy-momentum tensor which cancelled the usual, direct matter terms (4.7) to leave as final combined gravitational field in the special Kinnersley case $(4.16),(4.17)$ the net result

$$
\left[h_{\mu \nu}^{\prime}(x)\right]^{K}=\left[h_{\mu \nu}^{(\mathrm{har})}\right]^{K}+\partial_{\mu} \xi_{\nu}^{K}+\partial_{\nu} \xi_{\mu}^{K}=2 G \frac{m\left(s_{R}\right)}{\rho_{R}} k_{\mu} k_{\nu}
$$

Because of the specific algebraic structure $\propto k_{\mu} k_{\nu}$ of the last right-hand side of equation (4.30), the gravitational field $h_{\mu \nu}^{\prime K}$ is easily verified not to contain any physical $1 / r$ gravitational waves at (future null) infinity. This can be seen either by taking the usual transverse-traceless (TT) projection of the spatial components in some fixed reference frame [using $k_{i}=n_{i}+O(1 / r)$ ], or by checking, from the derivatives given in section 2 , that, at the $1 / \rho$ level, $\partial_{\alpha \beta} h_{\mu \nu}^{\prime K} \propto k_{\alpha} k_{\beta} k_{\mu} k_{\nu}$ which implies the vanishing of the linearized Riemann tensor at the $1 / \rho$ level. [A more complete calculation, first performed by Kinnersley [1], shows that the linearized Weyl tensor of (4.30) is of order $\rho^{-3}$ at infinity, while its linearized Ricci tensor, proportional to $T_{\mu \nu}$, is of order $\left.\rho^{-2}\right]$.

The non radiative result (4.30) has been derived in the special case where the anisotropy of the photon flux $\varepsilon(s, n)$ is purely dipolar, equations (4.16), (4.17). Let us briefly discuss the generic case where $\varepsilon(s, n)$ contains more general anisotropies. Using the notation (4.14), we can always write

$$
\varepsilon(s, n)=\varepsilon^{K}(s, n)+\sum_{\ell=2}^{\infty} \varepsilon_{\ell}(s, n)
$$


keeping unchanged the definition (4.16), and the result (4.17) which followed from the general balance equations (3.13). Let us then define $\sigma(s, n)$ as the unique solution of the differential equation

$$
D_{0} \sigma(s, n)=-4 G \varepsilon(s, n)
$$

which reduces to the constant $4 G m\left(s_{0}\right)$ when $s \leq s_{0}$ (i.e. before $\varepsilon(s, n)$ turns on). Here $D_{0}$ denotes the value at $B=0$ of the differential operator (4.12). The (local) existence and uniqueness of $\sigma$ follow from the fact that, when written explicitely in terms of $s$ and two angular coordinates $\left(\theta_{A}\right)=(\theta, \varphi)$ on the sphere, $D_{0}=\partial / \partial s+v^{A}\left(s, \theta_{B}\right) \partial / \partial \theta_{A}$ is a first-order linear operator. Given initial data for $\mathcal{L}$ in the past, and the monopole and dipole parts, $\varepsilon_{0}$ and $\varepsilon_{1}$, for all values of $s$ one can solve uniquely for the time evolution of $\mathcal{L}$ (via equations (3.13)) and then for $\sigma(s, n)$. The linearity of equation (4.32) means that its solution reads $\sigma=\sigma^{K}+\sigma^{\text {rad }}$, where $\sigma^{K}$ is the "Kinnersley" piece (4.19) and where $\sigma^{\mathrm{rad}}$ denotes the solution of $D_{0} \sigma^{\mathrm{rad}}=-4 G \sum_{\ell \geq 2} \varepsilon_{\ell}$.

From the explicit expression (4.12), one checks that, when expanding $\sigma^{\text {rad }}$ in orbital multipoles $\sigma_{\ell}^{\mathrm{rad}} \propto \widehat{n}_{\alpha_{1} \ldots \alpha_{\ell}}$, the $\ell=0$ and $\ell=1$ pieces of $D_{0} \sigma^{\mathrm{rad}}$ are entirely expressible in terms of $\sigma_{0}^{\mathrm{rad}}$ and $\sigma_{1}^{\mathrm{rad}}$ (without the presence of a term $\left.\propto \sigma_{2}^{\mu \nu} \dot{u}_{\mu} n_{\nu}\right)$. As the source term for $D_{0} \sigma^{\text {rad }}$ has only multipoles of order $\geq 2$, we conclude that $\sigma_{0}^{\text {rad }}=\sigma_{1}^{\text {rad }} \equiv 0$, i.e.

$$
\sigma(s, n)=4 G m(s)+\sum_{\ell=2}^{\infty} \sigma_{\ell}(s, n)
$$

where $\sigma_{2}+\sigma_{3}+\cdots$ is driven by $\varepsilon_{2}+\varepsilon_{3}+\cdots$.

Using as above $\sigma$ to define the gauge transformation (4.9), we find from equation (4.11) that the total gauge-transformed gravitational field reads

$$
h_{\mu \nu}^{\prime}=h_{\mu \nu}^{(\mathrm{har})}+\partial_{\mu} \xi_{\nu}+\partial_{\nu} \xi_{\mu}=\left[h_{\mu \nu}^{\prime}\right]^{K}+h_{\mu \nu}^{(p) \mathrm{rad}}
$$

where $\left[h_{\mu \nu}^{\prime}\right]^{K}$ is the non-radiative field (4.30) and 


$$
\begin{gathered}
h_{\mu \nu}^{(p) \mathrm{rad}}= \\
A C_{B=0}\left\{\square _ { R } ^ { - 1 } \left(\rho _ { R } ^ { B - 3 } \left\{\sigma^{\mathrm{rad}}\left[\frac{B}{3} \Delta_{\mu \nu}+\frac{B-2}{2}\left(n_{\mu} u_{\nu}+n_{\nu} u_{\mu}\right)+(B-3) \widehat{n}_{\mu \nu}\right]\right.\right.\right. \\
\left.\left.\left.+\frac{1}{2} \frac{\partial \sigma^{\mathrm{rad}}}{\partial n_{\alpha}}\left[\left(\Delta_{\alpha \mu}-n_{\alpha} n_{\mu}\right) k_{\nu}+\left(\Delta_{\alpha \nu}-n_{\alpha} n_{\nu}\right) k_{\mu}\right]\right\}\right)\right\}
\end{gathered}
$$

From formula (4.24), which can be compactly stated as

$$
A C_{B=0}\left\{\square_{R}^{-1}\left[\rho_{R}^{B-3} H\left(s_{R}(x), n\right)\right]\right\}=\rho_{R}^{-1} \Delta_{n}^{-1} H\left(s_{R}(x), n\right),
$$

where $\Delta_{n}$ is the Laplacian on the unit sphere, and $\Delta_{n}^{-1}$ its inverse acting on zero-mean functions on the sphere, we get $h_{\mu \nu}^{(p) \text { rad }}=\rho_{R}^{-1} U_{\mu \nu}\left(s_{R}, n\right)$ where $U_{\mu \nu}(s, n)$ is some explicit functional of $\sigma^{\mathrm{rad}}(s, n)$, which depends only on the values of $\sigma^{\text {rad }}$ for the same value of the proper time $s$. This computation has been done explicitly in the case of a straight worldline $\mathcal{L}$ in Ref. [10, see equations (2.20) there. In particular, the TT projection of equation (2.20c) there [in which only the last term $\propto\left(n_{L-2} \Pi_{i j L-2}\right)^{\text {TT }}$ survives] show that the $1 / r$ gravitational wave amplitude radiated at infinity is non zero as soon as $\sigma^{\text {rad }}(n)$ contains a non-zero multipole of order $\ell \geq 2$.

The conclusion is that, as soon as the photon flux function $\varepsilon(s, n)$ contains an anisotropy of multipole order $\ell \geq 2$, it will drive such terms in $\sigma^{\mathrm{rad}}(s, n)$ which will, through equations $(4.35),(4.36)$, radiate genuine $1 / r$ gravitational waves at future null infinity'?

\section{Conclusion}

After having shown the mathematical and physical $\left(\forall x \in \mathbb{R}^{4}, \partial_{\nu} T^{\mu \nu}(x)=0\right)$ consistency of the definition of massive point-like photon rockets in special relativity, we have studied the retarded linearized gravitational field that they generate. The crucial point is that the source of the gravitational radiation is the sum of the stress-energy tensor of the matter and of that of the outgoing flux of photons. In fact, the basic source of time-dependence in the system is the photon flux function $\varepsilon(s, n)$. The acceleration of the matter

\footnotetext{
${ }^{6}$ Note that in the limiting case of an infinitely massive photon rocket, $\mathcal{L}$ becomes straight and $\sigma^{\mathrm{rad}}(s, n)=-4 G \int_{-\infty}^{s} d s^{\prime} \sum_{\ell>2} \varepsilon_{\ell}\left(s^{\prime}, n\right)$, so that we can directly use the results of 10 to relate the multipoles of the gravitational wave at infinity to the multipoles of order $\ell \geq 2$ in $\varepsilon(s, n)$.
} 
is a derived quantity, computable from the monopolar and dipolar pieces in the spherical harmonics expansion of $\varepsilon(s, n)$ : equations (3.13). We have proven by an explicit calculation of the retarded potential generated by the total stress-energy tensor $T^{\mu \nu}=T_{(m)}^{\mu \nu}+T_{(p)}^{\mu \nu}$ that the amount of gravitational radiation at infinity is driven by the anisotropies of order $\ell \geq 2$ in the photon flux $\varepsilon(s, n)$. On the other hand, the coherent addition of the gravitational waves emitted by the monopolar and dipolar pieces of the photon energytensor, and of the gravitational waves emitted by the accelerated (because of the recoil) matter, exhibits a cancellation leaving a time-dependent, but nonradiative gravitational field (4.30). This cancellation is not too surprising if we consider, as recalled above, that the photon flux function $\varepsilon(s, n)$ is the basic source of time-dependence, and therefore gravitational radiation, in the system. In view of the spin 2 of the graviton, it makes sense that only the anisotropies of order $\ell \geq 2$ in $\varepsilon(s, n)$ can radiate gravitational waves.

We have presented the calculation of the emission of gravitational radiation in gory details to make it clear that there is absolutely no incompatibility between the non-radiative character of the gravitational field generated by a purely dipolar photon flux anisotropy and the perturbation techniques currently used to compute the generation of gravitational radiation in general relativity. Some of the technicalities of our treatment (notably the use of analytic continuation techniques) have been forced upon us by the necessity to deal in a mathematically rigourous way with the retarded potentials generated by peculiar singular sources $\propto \rho_{R}^{-2}$ (which become infinite on a worldline and fall off rather slowly at infinity). A careless treatment could miss the delicate compensation between the gravitational radiation emitted by the Dirac-distributed matter energy tensor, and that emitted by some of the singular terms $\propto \rho^{-3}$ appearing as effective sources in the gauge-transformed photon-generated metric $h_{\mu \nu}^{(p)}+\partial_{\mu} \xi_{\nu}+\partial_{\nu} \xi_{\mu}$.

\section{Appendix}

To make contact with the literature let us remark that, as a by product of the calculations above, we have shown that the linearized metric

$$
g_{\mu \nu}(x)=\eta_{\mu \nu}+h_{\mu \nu}^{\prime K}=\eta_{\mu \nu}+2 G \frac{m\left(s_{R}\right)}{\rho_{R}} k_{\mu} k_{\nu},
$$

satisfies everywhere in $\mathbb{R}^{4}$ (in the sense of distribution theory) the linearized Einstein equations

$$
R_{\mu \nu}^{\operatorname{lin}}-\frac{1}{2} R^{\operatorname{lin}} \eta_{\mu \nu}=8 \pi G \eta_{\nu \lambda} T_{\mu}^{\lambda}
$$


with $T_{\mu}^{\nu}$ given by equations (3.1)-(3.3).

Remarking now that (A.1) is of the Kerr-Schild form [12], and remembering that Gürses and Gürsey [13] have shown that all the nonlinear terms cancel when Kerr-Schild metrics are inserted into the Einstein equations written in terms of a mixed energy-momentum tensor $T_{\mu}^{\nu}$, we see that we have rederived (in a roundabout way) the result of Kinnersley [1], namely that the metric (A.1) is, outside the worldline $\mathcal{L}$, an exact solution of Einstein equations with source $T_{(p) \mu}^{\nu}=(4 \pi)^{-1} \rho_{R}^{-2} \varepsilon^{K}\left(s_{R}, n\right) k_{\mu} k^{\nu}$. Note that, in the exact, nonlinear framework of Einstein's equations, it makes no sense to write down $\delta$-like source terms, and therefore to say that the exact Kinnersley metric describes a point-like photon rocket. This is why, in the text, we consistently considered only linearized gravity. 


\section{References}

[1] Kinnersley W 1969 Phys. Rev. 1861335.

[2] Kramer D, Stephani H, MacCallum M and Herlt E 1980 Exact solutions of Einstein's field equations (Cambridge: Cambridge University Press).

[3] Bonnor W B 1994 Class. Quantum Grav. 112007.

[4] Schwartz L 1966 Théorie des distributions (Paris: Hermann).

[5] Hornby A S 1974 Oxford's advanced learner's dictionary of current English (Oxford: Oxford University Press).

[6] Blanchet L and Damour T 1986 Phil. Trans. R. Soc. Lond. A 320379.

[7] Blanchet L and Damour T 1988 Phys. Rev. D 371410.

[8] Blanchet L and Damour T 1989 Ann. Inst. H. Poincaré 50377.

[9] Damour T and Iyer B R 1991 Ann. Inst. H. Poincaré 54115.

[10] Blanchet L and Damour T 1992 Phys. Rev. D 464304.

[11] Chown M 1994 New Scientist 143 No 1943 ( 17 September 1994 ) p.15.

[12] Kerr R P and Schild A 1965 Proc. Symp. Appl. Math. 17199.

[13] Gürses M and Gürsey F 1975 J. Math. Phys. 162385. 